\title{
A Note on the Rosenbrock Procedure*
}

\author{
By T. D. Bui
}

\begin{abstract}
To be useful for extremely stiff systems of ordinary differential equations, $A$-stability and a maximally damped condition as $\lambda h \rightarrow-\infty$ (i.e., $L$-stability) are desirable. This paper investigates the condition of $L$-stability for a class of Runge-Kutta methods known as the Rosenbrock procedure. This procedure requires only one computation of a Jacobian matrix per step of integration. $L$-stable Rosenbrock methods up to order four are derived.
\end{abstract}

1. Introduction. In previous papers [5], [6] , some $A$-stable and $L$-stable methods for the numerical integration of stiff systems of ordinary differential equations were developed based on the Rosenbrock procedure [14]. The conditions of $A$-stability and $L$-stability require that the proposed methods must be implicit in nature. Butcher, Ehle and Bickart have investigated the problem of implementing fully implicit RungeKutta methods. Ehle [9] has shown that $R$-stage fully implicit Runge-Kutta methods of order $2 R$ developed in [3] are all $A$-stable. However, there is an impediment to using fully implicit Runge-Kutta methods. It is that when an $R$-stage method is applied to a system of $n$ stiff differential equations, then a system of $n R$ nonlinear algebraic equations will have to be solved at each time-step by some scheme of iteration. If the Newton method, or one of its variations, is used to solve these nonlinear systems, then it is necessary to solve $n R$ linear algebraic equations at each iteration-step within each time-step. Butcher [4] and Bickart [2] have developed separately different procedures to cut down the number of multiplications involved in the solution of the system of nonlinear algebraic equations resulting from an $R$-stage fully implicit RungeKutta method. However, fully implicit Runge-Kutta methods still suffer from many practical disadvantages and in practice they are not comparable with semi-implicit Runge-Kutta or Rosenbrock procedures.

Semi-implicit Runge-Kutta processes have been studied by $\mathrm{N} \phi$ rsett [12], N $\phi$ rsett and Wolfbrandt [13] and Alexander [1] for the stability conditions and attainable orders. Many $A$-stable and $L$-stable methods based on semi-implicit formulas were proposed in [12] and strongly stable methods based on diagonally implicit formulas were developed in [1]. However, when these methods are applied to a set of $n$ stiff differential equations, it is required to solve an $n$-dimensional system of nonlinear algebraic equations. The Rosenbrock procedure, which does not require the solution of nonlinear equations, has been investigated in [5], [6], [7], [10]. The implementation of this procedure requires only the solution of linear systems of algebraic equations, a much

Received August 14, 1975.

AMS (MOS) subject classifications (1970). Primary 65 L05.

* This work was supported in part by the National Research Council of Canada. 
simpler task compared to the first two approaches. In the following section, we will investigate some properties of the Rosenbrock procedure with respect to stability and attainable orders.

2. Conditions for $L$-Stability in Rosenbrock Procedure. We are concerned with the numerical solutions of the system of differential equations

$$
d y / d x=f(y), \quad x>x_{0}, \quad y\left(x_{0}\right)=y_{0},
$$

where $x \in R, y(x) \in R^{n}, y_{0} \in R^{n}$ and $f: R^{n} \rightarrow R^{n}$ is assumed to be analytic in the neighborhood of $y_{0}$. The system of differential equations (1) is called stiff in an interval $I$, if $\forall x \in I$ the eigenvalues $\lambda_{i}(x)$ of the Jacobian $\delta f / \delta y$ satisfy the following conditions:

(i) Real $\lambda_{i}(x)<0, i=1,2, \ldots, n$,

(ii) $\max _{x} \mid$ Real $\lambda_{i}(x)\left|\gg \min _{x}\right| \operatorname{Real} \lambda_{i}(x) \mid$.

The Rosenbrock procedure for solving Eq. (1) is defined by

$$
\begin{gathered}
y_{m+1}=y_{m}+h \sum_{i=1}^{s} w_{i} k_{i}, \\
k_{i}=f\left(y_{m}+h \sum_{j=1}^{i-1} a_{i j} k_{j}\right)+d_{i} h \frac{\delta f}{\delta y}\left(y_{m}+h \sum_{j=1}^{i-1} b_{i j} k_{j}\right) k_{i} .
\end{gathered}
$$

Definition 1. A method is called $A$-stable in the sense of Dahlquist [8] if and only if $\left|y_{m+1} / y_{m}\right|=c \leqslant 1$ when the method is applied with any positive step-size $h$ to the test equation

$$
d y / d x=\lambda y,
$$

where $\lambda$ is a complex constant with negative real part.

Definition 2 [11]. A method is called $L$-stable if it is $A$-stable and $c \rightarrow 0$ as $\lambda h \rightarrow-\infty$.

THEOREM 1. When an s-stage Rosenbrock procedure is applied to the test equation (3), the procedure reduces to a rational function in $q=\lambda h$

$$
\frac{y_{m+1}}{y_{m}}=\frac{\sum_{i=0}^{r} \alpha_{i} q^{i}}{\prod_{i=1}^{s}\left(1-d_{i} q\right)}=R_{r, s}(q) \quad \text { where } r \leqslant s \text { and } \alpha_{0}=1 \text {. }
$$

Proof: By direct substitution of Eq. (3) into (2), it is straightforward (but tedious) to prove Theorem 1 by noting that $\left(a_{i j}\right)$ is a matrix with all the elements in the diagonal and upper triangular part equal to zero.

THEOREM 2. The order of an L-stable, s-stage Rosenbrock procedure is at most s.

Proof. $\mathrm{N} \phi$ rsett [12] has proved that the maximum order of an $\mathrm{N}$-approximation (i.e., $R_{m, n}(q)$ with only real poles) to $\exp (q)$ is $m+1$. It is easy to prove that $R_{r, s}(q)$ given by (4) is an $N$-approximation to $\exp (q)$. Therefore, the maximum order of an $L$-stable, $s$-stage Rosenbrock procedure is $r+1 \leqslant s$. 
THEOREM 3. When $d_{i}=d$ (for all $i$ ) is the inverse of one of the roots of the Laguerre polynomial of degree $s\left(\right.$ i.e., $\left.L_{s}(x)=0\right)$, then the corresponding Rosenbrock procedure (Eqs. (2)) has a rational approximation $R_{r, s}$ with $r=s-1$ and whose order is $s$.

Proof. $R_{r, s}(q)$ is said to be rational approximation of order $r$ to $\exp (q)$ if

$$
R_{r, s}(q)=\exp (q)+O\left(q^{r+1}\right), \quad|q| \rightarrow 0 \text { and } r \geqslant 0 .
$$

Using Eq. (4) and expanding $\exp (q)$ in powers of $q$, we have

$$
\frac{\sum_{i=0}^{r} \alpha_{i} q^{i}}{\sum_{i=0}^{s} \beta_{i} q^{i}}=\sum_{i=0}^{r} \frac{1}{i !} q^{i}+O\left(q^{r+1}\right), \quad \alpha_{0}=\beta_{0}=1
$$

where $\Pi_{i=1}^{s}\left(1-d_{i} q\right)=\Sigma_{i=0}^{s} \beta_{i} q^{i}$.

Equating terms of the same power of $q$ from both sides, we have

$$
\alpha_{i}=\sum_{j=0}^{i} \frac{\beta_{j}}{(i-j) !} \text { for } i=0,1, \ldots, r
$$

The condition of $L$-stability requires that

$$
\sum_{i=0}^{s} \frac{\beta_{i}}{(s-i) !}=0 .
$$

Since $\Sigma_{i=0}^{s} \beta_{i} q^{i}=\Pi_{i=1}^{s}\left(1-d_{i} q\right)=(1-d q)^{s}$, then the binomial theorem gives $\beta_{i}=$ $(-1)^{i}\left(\begin{array}{l}s \\ i\end{array}\right) d^{i}$ and Eq. (5) becomes

$$
\sum_{i=0}^{s} \frac{(-1)^{i}\left(\begin{array}{l}
s \\
i
\end{array}\right) d^{i}}{(s-i) !}=0 \quad \text { or } \quad \sum_{i=0}^{s} \frac{(-1)^{i}\left(\begin{array}{l}
s \\
i
\end{array}\right) d^{-i} s !}{i !}=0 .
$$

From the definition of Laguerre polynomial of degree $s$, we have $L_{s}(1 / d)=0$.

For the Rosenbrock procedure, whose rational approximation is given by Eq. (4), the condition of $L$-stability (Definition 2 ) is equivalent to the following two conditions:

(i) $\left|y_{m+1} / y_{m}\right| \leqslant 1$ on $\operatorname{Real}(\lambda h)=0$.

(ii) $y_{m+1} / y_{m}$ has lower numerator degree than denominator degree in $q=\lambda h$.

Condition (ii) is achieved when Theorem 3 is satisfied. Thus, Rosenbrock procedures satisfying Theorem 3 and condition (i) simultaneously are $L$-stable methods with highest possible orders. The derivation of a specific Rosenbrock method requires the solution of a set of the order equations. These equations are obtained by matching terms of the same order in the Taylor series expansions of the Rosenbrock procedure.

\section{Some $L$-Stable Rosenbrock Methods.}

Two-Stage Method of Order Two. In this case, $L_{2}(x)=0$ has two roots. The inverses of these roots are $1+1 / \sqrt{2}$ and $1-1 / \sqrt{2}$. Theorem 3 states that the parameter $d$ must have one of these two values. Furthermore, we can prove that $\left|R_{1,2}(q)\right| \leqslant 1$ on real $(q)=0$ for both values of $d$. Thus, they satisfy the condition of $L$-stability. Cash [7] and Rosenbrock [14] choose $d=1+1 / \sqrt{2}$ and $d=1-1 / \sqrt{2}$, respectively, for the development of their $L$-stable methods. 
Three-Stage Method of Order Three. For $L_{3}(x)=0$, we have three roots: 0.415 $7745568,2.2942803606$ and 6.2899450829 . However, only the second root satisfies the condition that $\left|R_{2,3}(q)\right| \leqslant 1$ on real $(q)=0$. Thus, the condition of $L$-stability is achieved only for $d=0.4358665216$. We have proved that the following scheme is $L$-stable:

$$
\begin{aligned}
w_{1} & =0 \\
w_{2} & =w_{3}=1 / 2 \\
a_{21} & =-0.5096436824 \\
a_{31} & =0.3270258661 \\
a_{32} & =0.3108847731 \\
b_{i j} & =0 \text { for } i=2(1) 3, j=1(1) i-1
\end{aligned}
$$

Alexander [1] has investigated this case for diagonally implicit Runge-Kutta methods. The diagonally implicit Runge-Kutta method is defined by Eqs. (2) with Eq. (2b) replaced by

$$
k_{i}=f\left(y_{m}+h \sum_{j=1}^{s} a_{i j} k_{j}\right) \quad \text { for } i=1,2, \ldots, s
$$

where

$$
\left(a_{i j}\right)=\left[\begin{array}{ccccr}
a & 0 & 0 & \ldots & 0 \\
a_{21} & a & 0 & \ldots & 0 \\
a_{31} & a_{32} & a & \cdots & 0 \\
----- & ------ \\
a_{s 1} & a_{s 2} & a_{s 3} & \cdots & a
\end{array}\right],
$$

i.e., $a_{i i}=a$ and $a_{i j}=0$ for all $j>i$.

Comparing Eqs. (2b) and (6), we see that the Rosenbrock procedure can be considered as a linearization of the diagonally implicit procedure.

Four-Stage Method of Order Four. Since $L_{4}(x)=0$ has four roots: 0.322547 $6896,1.7457611012,4.5366202969,9.3950709123$, the necessary condition for $L$-stability requires that the parameter $d$ should have one of the following values: $d_{1}=3.100316735, d_{2}=0.5728160625, d_{3}=0.2204284103, d_{4}=0.106$ 4387921 . However, it is easy to prove that only $d_{2}$ satisfies the sufficient condition for $L$-stability (i.e., $\left|R_{3,4}(q)\right| \leqslant 1$ ). Using this value for the parameter $d$, a fourth order $L$-stable method was developed in [5] which also minimizes the error constant. This method is defined by the following set of parameters:

$$
\begin{aligned}
& w_{1}=0.9451564786, \\
& w_{2}=0.341323172, \\
& w_{3}=0.5655139575, \\
& w_{4}=-0.8519936081, \\
& a_{21}=-0.5, \\
& a_{31}=-0.1012236115, \\
& a_{32}=0.9762236115, \\
& a_{41}=-0.3922096763,
\end{aligned}
$$




$$
\begin{aligned}
a_{42} & =0.7151140251, \\
a_{43} & =0.1430371625, \\
b_{i j} & =0 \quad \text { for } i=2(1) 4, j=1(1) i-1 .
\end{aligned}
$$

4. Conclusions. New $L$-stable Rosenbrock processes of higher order can be derived by using theorems presented in Section 2 . The only drawback for implementing Rosenbrock processes is the problem of evaluating the Jacobian matrix at every timestep. We are investigating procedures in which the Jacobian matrix is evaluated only once at the beginning of an interval of $x$, then the same value is used again for the whole interval.

Department of Computer Science

Concordia University

Montreal, Quebec, Canada

1. R. ALEXANDER, “Diagonally implicit Runge-Kutta methods for stiff O.D.E.'s," SIAM J. Numer. Anal., v. 14, 1977, pp. 1006-1021.

2. T. A. BICKART, “An efficient solution process for implicit Runge-Kutta methods," SIAM J. Numer. Anal., v. 14, 1977, pp. 1022-1027.

3. J. C. BUTCHER, "Implicit Runge-Kutta processes," Math. Comp., v. 18, 1964, pp. 50-64.

4. J. C. BUTCHER, "On the implementation of implicit Runge-Kutta methods," BIT, v. 16, 1976, pp. 237-240.

5. T. D. BUI, "On an $L$-stable method for stiff differential equations," Inf. Proc. Letters, v. 6, 1977, pp. 158-161; see also "Erratum," Inf. Proc. Letters. (To appear.)

6. T. D. BUI, "Some $A$-stable and $L$-stable methods for the numerical integration of stiff ordinary differential equations," J. Assoc. Comput. Mach. (To appear.)

7. J. R. CASH, "Semi-implicit Runge-Kutta procedures with error estimates for the numerical integration of stiff systems of ordinary differential equations," J. Assoc. Comput. Mach., v. 23, 1976, pp. 455-460; see also: T. D. BUI, "Errata and comments on a paper by J. R. Cash," J. Assoc. Comput. Mach., v. 24, 1977, p. 623.

8. G. G. DAHLQUIST, "A special stability problem for linear multistep methods," BIT, v. 3, 1963, pp. 27-43.

9. B. L. EHLE, "High order $A$-stable methods for the numerical solution of differential equations," $B I T$, v. 8, 1968 , pp. 276-278.

10. C. F. HAINES, "Implicit integration processes with error estimates for the numerical solution of differential equations," Comput. J., v. 12, 1968, pp. 183-187.

11. J. D. LAMBERT, Computational Methods in Ordinary Differential Equations, Wiley, New York, 1973.

12. S. P. NФRSETT, Semi-Explicit Runge-Kutta Methods, Math. and Comp. Report 6/74, Dept. of Math., University of Trondheim, Norway, 1974.

13. S. P. N $\varphi R S E T T \&$ A. WOLFBRANDT, "Attainable order of rational approximations to the exponential function with only real poles," $B I T$, v. 17, 1977, pp. 200-208.

14. H. H. ROSENBROCK, "Some general implicit processes for the numerical solution of differential equations," Comput. J., v. 5, 1963, pp. 329-330. 